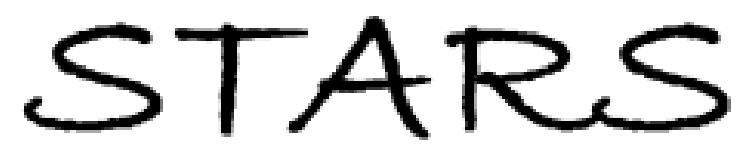

University of Central Florida

STARS

$1-1-2009$

\title{
Photoresponse in large area multiwalled carbon nanotube/ polymer nanocomposite films
}

\author{
Paul Stokes \\ University of Central Florida \\ Liwei Liu \\ University of Central Florida \\ Jianhua Zou \\ University of Central Florida
}

Lei Zhai

University of Central Florida

Qun Huo

University of Central Florida

Find similar works at: https://stars.library.ucf.edu/facultybib2000

University of Central Florida Libraries http://library.ucf.edu

See next page for additional authors

This Article is brought to you for free and open access by the Faculty Bibliography at STARS. It has been accepted for inclusion in Faculty Bibliography 2000s by an authorized administrator of STARS. For more information, please contactSTARS@ucf.edu.

\section{Recommended Citation}

Stokes, Paul; Liu, Liwei; Zou, Jianhua; Zhai, Lei; Huo, Qun; and Khondaker, Saiful I., "Photoresponse in large area multiwalled carbon nanotube/polymer nanocomposite films" (2009). Faculty Bibliography 2000s.

2185.

https://stars.library.ucf.edu/facultybib2000/2185

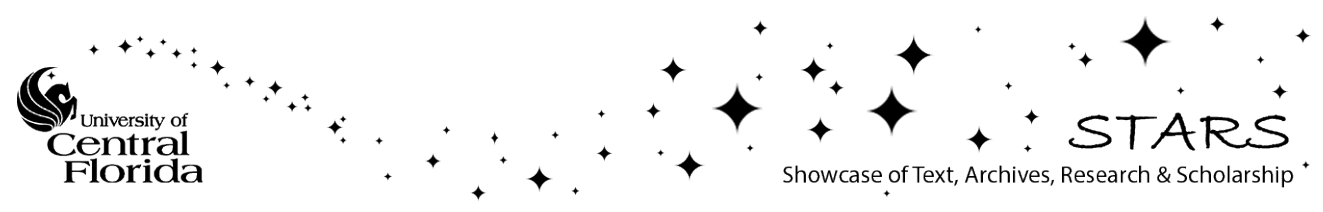




\section{Authors}

Paul Stokes, Liwei Liu, Jianhua Zou, Lei Zhai, Qun Huo, and Saiful I. Khondaker 


\section{Photoresponse in large area multiwalled carbon nanotube/polymer nanocomposite films}

Cite as: Appl. Phys. Lett. 94, 042110 (2009); https://doi.org/10.1063/1.3075957

Submitted: 07 November 2008 . Accepted: 05 January 2009. Published Online: 30 January 2009

Paul Stokes, Liwei Liu, Jianhua Zou, Lei Zhai, Qun Huo, and Saiful I. Khondaker

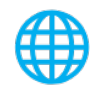

\section{ARTICLES YOU MAY BE INTERESTED IN}

High performance multiwall carbon nanotube bolometers

Journal of Applied Physics 108, 084305 (2010); https://doi.org/10.1063/1.3492633

Suspending single-wall carbon nanotube thin film infrared bolometers on microchannels Applied Physics Letters 94, 163110 (2009); https://doi.org/10.1063/1.3124651

Fully printed flexible carbon nanotube photodetectors

Applied Physics Letters 110, 123105 (2017); https://doi.org/10.1063/1.4978935

\section{Applied Physics Letters}

Mid-IR and THz frequency combs special collection 


\title{
Photoresponse in large area multiwalled carbon nanotube/polymer nanocomposite films
}

\author{
Paul Stokes, ${ }^{1,2}$ Liwei Liu, ${ }^{1,2}$ Jianhua Zou, ${ }^{1,3,4}$ Lei Zhai, ${ }^{1,3,4}$ Qun Huo, ${ }^{1,3,4}$ and \\ Saiful I. Khondaker ${ }^{1,2, a)}$ \\ ${ }_{1}^{1}$ Nanoscience Technology Center, University of Central Florida, Orlando, Florida 32826, USA \\ ${ }^{2}$ Department of Physics, University of Central Florida, Orlando, Florida 32826, USA \\ ${ }^{3}$ Department of Mechanical, Materials and Aerospace Engineering, University of Central Florida, \\ Orlando, Florida 32826, USA \\ ${ }^{4}$ Department of Chemistry, University of Central Florida, Orlando, Florida 32826, USA
}

(Received 7 November 2008; accepted 5 January 2009; published online 30 January 2009)

\begin{abstract}
We present a near IR photoresponse study of large area multiwalled carbon nanotube/ poly(3-hexylthiophene)-block-polystyrene polymer (MWNT/P3HT-b-PS) nanocomposite films for different loading ratio of MWNT into the polymer matrix. We show that the photocurrent strongly depends on the position of the laser spot with maximum photocurrent occurring at the metal-film interface. In addition, compared to the pure MWNT film, the photoresponse is much larger in the MWNT/polymer composite films. The time constant for the photoresponse is slow and varies between 0.6 and $1.2 \mathrm{~s}$. We explain the photoresponse by Schottky barrier modulation at the metal-film interface. (C) 2009 American Institute of Physics. [DOI: 10.1063/1.3075957]
\end{abstract}

Carbon nanotubes (CNTs) are considered to be promising building blocks for nanoelectronic and optical devices due to their special geometry, high electrical conductivity, and exceptional mechanical and optical properties. ${ }^{1-3}$ Recently, the photoresponse of CNTs [both in visible and near infrared (NIR) regime] has generated considerable debate in terms of whether the photoresponse is (i) due to photon induced charge carrier (excitonic), (ii) due to heating of the CNT network (bolometric), or (iii) caused by photodesorption of oxygen molecules at the surface of the CNT. In addition, the role of the metal-CNT contact effect on the photoresponse has also been debated. In individual semiconducting single-walled carbon nanotube (SWNT) field effect transistor (FET) devices, Freitag et $\mathrm{al}^{4}{ }^{4}$ and Qiu et al. ${ }^{5}$ explained the photoresponse in the NIR regime using an exciton model. Chen et al. ${ }^{6}$ showed that in individual SWNT FET devices, the reduction in conductivity upon UV illumination is due to the desorption of molecular oxygen from the CNT surface, which causes a reduction in hole carriers. In contrast, for a large area SWNT film suspended in vacuum, it was argued by Itkis et al. ${ }^{7}$ that the NIR photoresponse was due to a bolometric effect, a change in conductivity due to heating of the SWNT network. In a microscopic SWNT film, Levitsky and Euler ${ }^{8}$ also found molecular photodesorption to be responsible for a change in conductivity upon NIR illumination. Other studies in macroscopic SWNT film show that the maximum photoresponse occurs at the CNT filmmetal interface and that the response varies with the position of laser illumination. ${ }^{9-12}$ This effect has been explained using exciton model where CNT-metal interface helps to separate electron and holes to induce a photovoltage. A recent study has shown that a mutiwalled carbon nanotube (MWNT) film also displays a positional dependent photocurrent. ${ }^{13} \mathrm{Al}-$ though there are many studies for the photoresponse in individual CNTs and CNT films, there are almost no studies on

\footnotetext{
${ }^{\text {a) }}$ Author to whom correspondence should be addressed. Electronic mail: saiful@mail.ucf.edu.
}

CNT/polymer nanocomposites. Nanocomposites may provide enhanced functionality as they form the basis of advanced materials with tailored properties. Their ease of processibility in solution, multifunctionality, flexibility, and low cost of fabrication make them attractive candidates for large area optoelectronic devices. ${ }^{14-16}$

In this letter, we present a NIR photoresponse study of MWNT/poly(3-hexylthiophene)-block-polystyrene (MWNT/ P3HT-b-PS) polymer nanocomposite film for different loading ratio of MWNT in polymer matrix. We found that upon NIR illumination, the photocurrent either increases, remains almost zero, or decreases based on the position of the illumination with respect to electrodes with maximum photocurrent occurring when illuminated at the metal-film interface. The photoresponse [(light current-dark current)/dark current] or $\left[\left(I_{\text {light }}-I_{\text {dark }}\right) / I_{\text {dark }}\right]$ is up to $164 \%$ when MWNT is embedded into P3HT-b-PS, while for pure MWNT film it is only $4 \%$. The time constant of the photoresponse is rather slow, between 0.6 and $1.2 \mathrm{~s}$. We explain the contact dependence photoresponse using excitonic picture and discuss reasons for the slow time response.

MWNT/P3HT-b-PS composites were prepared following our recently developed technique. ${ }^{15}$ Solutions with MWNT loading ratios of $0.25 \%, 0.50 \%, 0.75 \%, 1.00 \%, 1.25 \%$, $1.50 \%, 2.00 \%, 3.00 \%, 5.00 \%$, and $10.00 \%$ were prepared. In addition a pure P3HT-b-PS film and a pure MWNT film were also prepared for control experiments. The solution was then drop cast onto the glass substrate using a draw-down bar, and the solvent was allowed to evaporate in a fume hood for a few hours. Resulting films were $\sim 40-60 \mu \mathrm{m}$ thick. Finally, $\sim 60 \mathrm{~nm}$ thick $\mathrm{Au}$ electrodes spaced $L \sim 3 \mathrm{~mm}$ and with width of $W \sim 20 \mathrm{~mm}$ were thermally evaporated using a shadow mask.

Figure 1(a) shows a cartoon of a final device and the electrical transport measurement setup. The room temperature dc transport measurements of the composite films were carried out using a standard two-probe technique both in dark and under illumination by a laser spot positioned at 

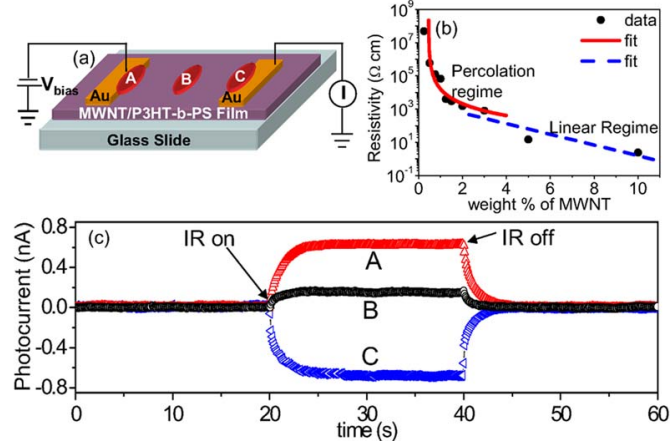

FIG. 1. (Color online) (a) Cartoon of the device and transport measurement setup. (b) Resistivity of the film vs weight percentage of MWNT in the polymer matrix. (c) Representative photocurrent as a function of time $(t)$ for $1.5 \%$ film under IR illumination at positions $\mathrm{A}, \mathrm{B}$, and $\mathrm{C}\left(V_{\text {bias }}=1 \mathrm{mV}\right)$.

three different locations [Fig. 1(a)]. A corresponds to illumination on the left electrode/film interface, $\mathrm{B}$ is between the electrodes in the middle of the sample, and $\mathrm{C}$ is the right electrode/film interface. The NIR photosource consists of a semiconductor laser diode with peak wavelength of $806 \mathrm{~nm}$ $(1.54 \mathrm{eV})$ driven by a Keithley 2400 . The spot size of the laser was approximately $10 \mathrm{~mm}$ long and $1 \mathrm{~mm}$ wide. The photo intensity is monitored with a calibrated silicon photodiode (Thorlabs S121 B). The power intensity of the laser is $\sim 5 \mathrm{~mW} / \mathrm{mm}^{2}$ at the distance it was placed from the sample $(\sim 2.5 \mathrm{~cm})$. Photocurrent was measured under small $V_{\text {bias }}$ $(1 \mathrm{mV})$ unless mentioned otherwise. Data were collected by means of LABVIEW interfaced with the data acquisition card and current preamplifier (DL instruments: Model 1211) capable of measuring sub-pA signal.

Figure 1(b) shows the resistivity of the sample for different loading ratios of the MWNT in P3HT-b-PS matrix measured in dark. By varying the fraction of MWNT added into the solution, the electrical resistivity can be controlled by several orders of magnitude. The resistivity of the films follows the standard percolation formula $\rho \propto\left(p-p_{c}\right)^{-\alpha}$, where $p_{c}$ is the critical volume fraction and $\alpha$ is the critical exponent. From the fit to our data, we obtain $p_{c}=0.45 \%$ and $\alpha$ $=1.95$. For large concentration of MWNT, the resistivity reduces linearly. The critical exponent we obtain is within reasonable agreement for what is expected from theoretical predictions. ${ }^{17}$

Figure 1(c) shows a typical photoresponse curve for one of our composite films (1.5\% MWNT) where we plot photocurrent as a function of time $(t)$ measured under a constant bias voltage of $1 \mathrm{mV}$ when the laser spot is positioned at $\mathrm{A}$, $\mathrm{B}$, and C. The IR source is turned on at $t=20 \mathrm{~s}$, left on for $20 \mathrm{~s}$, and then turned off at $t=40 \mathrm{~s}$. The current under no illumination (dark current) was subtracted from the photocurrent measurements. Three features can be noticed from this figure: (a) the photocurrent is positional dependent, (b) there is a large enhancement in the photocurrent at the metalfilm interface, and (c) the response time is rather slow. When illuminated at position A there is an increase in photocurrent. Position B also shows an increase in current but much smaller, whereas position $\mathrm{C}$ shows a decrease in photocurrent when illuminated by the IR source. Similar behavior of the photocurrent has been observed in all our samples with different MWNT loading ratios excluding the pure P3HT-b-PS film and the $0.25 \%$ MWNT loading ratio. For these two samples, under $1 \mathrm{mV}$ bias, signal to noise $(\mathrm{S} / \mathrm{N})$ ratio is ex-
TABLE I. Dark current, light current, photoresponse, and EQE for MWNT/ P3HT-b-PS nanocomposite films at position A.

\begin{tabular}{ccccc}
\hline \hline wt \% of MWNT & $I_{\text {dark }}(\mathrm{A})$ & $I_{\text {light }}(\mathrm{A})$ & $\Delta I / I_{\text {dark }}(\%)$ & $\mathrm{EQE}$ \\
\hline 0.5 & $1.27 \times 10^{-11}$ & $3.35 \times 10^{-11}$ & 164 & $6.4 \times 10^{-07}$ \\
0.75 & $5.26 \times 10^{-11}$ & $6.72 \times 10^{-11}$ & 27.8 & $4.5 \times 10^{-07}$ \\
1 & $1.07 \times 10^{-10}$ & $1.33 \times 10^{-11}$ & 24.3 & $8.0 \times 10^{-07}$ \\
1.25 & $1.78 \times 10^{-9}$ & $2.10 \times 10^{-9}$ & 18.0 & $9.9 \times 10^{-06}$ \\
1.5 & $3.16 \times 10^{-9}$ & $3.78 \times 10^{-9}$ & 18.8 & $1.8 \times 10^{-05}$ \\
2 & $6.59 \times 10^{-9}$ & $8.16 \times 10^{-9}$ & 24.2 & $4.9 \times 10^{-05}$ \\
3 & $1.18 \times 10^{-8}$ & $1.32 \times 10^{-8}$ & 11.9 & $4.3 \times 10^{-05}$ \\
5 & $1.11 \times 10^{-6}$ & $1.31 \times 10^{-6}$ & 19.1 & $6.5 \times 10^{-03}$ \\
10 & $3.14 \times 10^{-6}$ & $3.80 \times 10^{-6}$ & 21.0 & $2.0 \times 10^{-02}$ \\
100 & $4.65 \times 10^{-5}$ & $4.84 \times 10^{-5}$ & 4.10 & $5.9 \times 10^{-02}$ \\
\hline \hline
\end{tabular}

tremely low; therefore the photocurrent was not very reproducible. The $\mathrm{S} / \mathrm{N}$ ratio for $0.5 \%$ composite film was 21 while for all other samples it was $\sim 70$ or higher making the detection of photocurrent easier (see supplementary information containing other $\mathrm{S} / \mathrm{N}$ ratios and $I$ vs $t$ curves). ${ }^{18}$ Table I summarizes experimental data from all our samples, where we show dark current, light current, photoresponse, and external quantum efficiency (EQE) for different loading ratios of MWNT composite film at position A. The EQE at $\lambda$ $=806 \mathrm{~nm}$ at a fixed power of $5 \mathrm{~mW}$ was calculated using $\mathrm{EQE}=\left(R_{\lambda} / \lambda\right) \times 1240 \mathrm{~W} \mathrm{~nm} / \mathrm{A}$, where $R_{\lambda}$ is the responsivity defined by the photocurrent per watt of input power. As can be seen from this table, the photoresponse is $164 \%$ for $0.5 \%$ MWNT/P3HT-b-PS composite film, while it is only $4.1 \%$ for pure MWNT film. The stronger photoresponse in MWNT/ P3HT-b-PS films could be due to semiconducting nature of these films compared to pure MWNT film where metallic pathways dominate. We note that in SWNT-polycarbonate composite films studied by Pradhan et al., ${ }^{19}$ such photoresponse was also observed. However, the maximum photoresponse was only $5 \%$ and could be due to the illumination of IR around the middle part of their sample and that the effect of contact was not checked.

In order to examine the position dependent photocurrent further, we measured the current-voltage $(I-V)$ characteristics for all of our samples. Figure 2(a) shows a representative $I-V$ curve of a $0.5 \%$ MWNT composite film when illuminated at position $\mathrm{A}$, position $\mathrm{C}$, and in the dark. The dark $I-V$ curve runs directly though the origin, whereas when illuminated on positions A and $\mathrm{C}$, the $I-V$ curve is slightly shifted above or below the origin, respectively. At zero applied bias, there is about $+15 \mathrm{pA}$ current at $\mathrm{A}$ and $-15 \mathrm{pA}$ at $\mathrm{C}$. This is more clearly seen in the inset of Fig. 2(a). Very little or no photocurrent at zero bias was observed at position B. The zero bias photocurrent increases in magnitude at positions $\mathrm{A}$ and $\mathrm{C}$ for increasing MWNT loading ratio. It is therefore clear that the
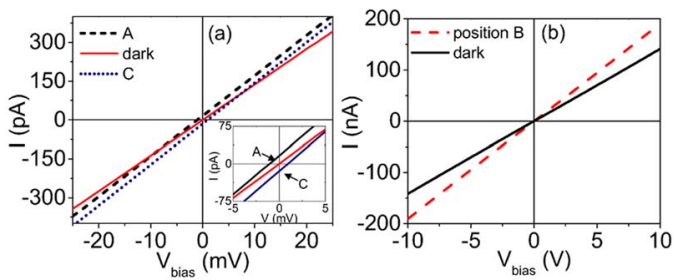

FIG. 2. (Color online) (a) Current-voltage characteristics of a 0.5 wt $\%$ MWNT composite film illuminated at positions A, C, and in the dark. Inset: zoomed in view around the origin. (b) Full range current-voltage characteristics in the dark and when illuminated at B. 


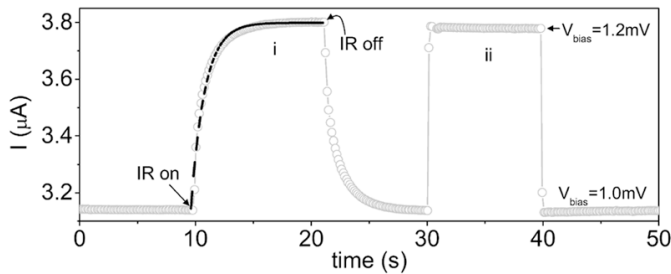

FIG. 3. (Color online) Current as a function of time $(t)$ for $1.5 \%$ film when (i) illuminated at position $\mathrm{A}$ and (ii) with a bias voltage spike in dark at $t$ $=30 \mathrm{~s}$, left on at $1.2 \mathrm{mV}$ for $10 \mathrm{~s}$, and then reduced to $1.0 \mathrm{mV}$ at $t=40 \mathrm{~s}$. The fit is shown as the dashed line, which gives a time constant of $1.2 \mathrm{~s}$.

finite current at zero bias must be caused by a photoinduced voltage related to metal composite film interface. It can also be seen from Fig. 2(a) that beyond certain critical positive applied bias, the photocurrent is always positive at all positions. This means that if one applies source drain bias beyond this voltage, the contact effect may become nonnoticeable. The critical voltage increases from 5 to $50 \mathrm{mV}$ from lowest to highest weight percent in our samples. This may be a measure of the Schottky barrier in our samples. Figure 2(b) shows the dark and light $I-V$ curve up to $10 \mathrm{~V}$ when the sample is illuminated at B. Here we do not see any offset at zero bias when illuminated.

We now examine the slow rise and decay of photocurrent when NIR source is switched on and off. Figure 3 is a plot of current as a function of time for $1.5 \%$ composite for two cases: (i) the IR is turned on at $t=10 \mathrm{~s}$, held on for $10 \mathrm{~s}$, and turned off at $t=20 \mathrm{~s}$ at position A with $V_{\text {bias }}=1 \mathrm{mV}$, and (ii) the bias is increased from 1.0 to $1.2 \mathrm{mV}$ at $t=30 \mathrm{~s}$ and then back to $1.0 \mathrm{mV}$ at $t=40 \mathrm{~s}$. When illuminated by the IR source the sample responds slowly until it reaches its steady state and slowly recovers to the dark state current when the laser is turned off. In contrast, the film responds almost instantaneously to a bias voltage switch. Therefore, the time constant indeed comes from the IR illumination and not a delay due to an $R$ - $C$-like circuit existing in the entire setup. The dynamic response to the IR source is well described by the exponential $I(t)=I_{\text {light }}+D \exp (-t / \tau)$, where $\tau$ is the time constant and $D$ is a scaling constant. From the fit in Fig. 3 we find the time constant to be $1.2 \mathrm{~s}$. The time constant measured for all our sample ranges from 0.6 to $1.2 \mathrm{~s}$ and does not show any clear pattern as we increase the loading ratio of MWNT.

All of the results presented here are consistent with the model of Schottky barrier modulation for photocurrent generation. ${ }^{10}$ In other words, when the laser light is illuminated at the interface, some energetic electrons overcome the tunnel barrier at the interface and fall into metal electrodes, leaving holes in the film. This causes an electron-hole separation at the interface and thereby creates a local electric field, whereas when the laser is shined in the middle electron hole pairs are created, however, the charge does not get separated, so the overall photovoltage is zero. We rule out both bolometric and molecular photodesorption because the positive and negative photoresponses at two different positions cannot be explained by these models. In addition, for bolometric response the time constant is expected to be in the
1-100 ms range. ${ }^{7}$ Recently, slow photoresponse in SWNT film has been explained by charge carrier diffusion due to the low mobility of the interconnected SWNT network compared to individual SWNT device. If charge carrier diffusion was responsible in our devices, we would have expected the time constant to decrease as we increase MWNT loading ratio due to increased mobility. Repeated measurements on several sets of samples did not reveal any correlation. Further experimental and theoretical work will be needed to explain the slow time constant in the nanocomposite films.

In conclusion, we present a NIR photoresponse study of large area MWNT/P3HT-b-PS films for different loading ratio of MWNT. We found that upon NIR illumination, the photocurrent is either positive, negative, or almost zero based on the position of the illumination with respect to electrodes. The photoresponse is up to $164 \%$ when MWNT is embedded into P3HT-b-PS, while for pure MWNT film it is only $4 \%$. The time constant of the photoresponse is rather slow, between 0.6 and $1.2 \mathrm{~s}$. Our results are consistent with the model of Schottky barrier modulation for photocurrent generation. This work shows promising route for the fabrication of large area low cost IR photo detectors and position sensitive detectors.

We thank Robert Peale for many useful discussions. This work is partially supported by US NSF under grants ECCS 0748091 and ECCS 0801924 to SIK, DMR 0746449 to LZ, and DMR 0552295, DMI 0506531 (NIRT) to QH.

${ }^{1}$ P. L. McEuen and J. Park, MRS Bull. 29, 272 (2004).

${ }^{2}$ P. Avouris, Z. H. Chen, and V. Perebeinos, Nat. Nanotechnol. 2, 605 (2007).

${ }^{3}$ R. H. Baughman, A. A. Zakhidov, and W. A. de Heer, Science 297, 787 (2002).

${ }^{4}$ M. Freitag, Y. Martin, J. A. Misewich, R. Martel, and Ph. Avouris, Nano Lett. 3, 1067 (2003).

${ }^{5}$ X. Qiu, M. Freitag, V. Perebeinos, and Ph. Avouris, Nano Lett. 5, 749 (2005).

${ }^{6}$ R. J. Chen, N. R. Franklin, J. Kong, J. Cao, T. W. Tombler, Y. Zhang, and H. Dai, Appl. Phys. Lett. 79, 2258 (2001).

${ }^{7}$ M. E. Itkis, F. Borondics, A. Yu, and R. C. Haddon, Science 312, 413 (2006).

${ }^{8}$ I. A. Levitsky and W. B. Euler, Appl. Phys. Lett. 83, 1857 (2003).

${ }^{9}$ C. A. Merchant and N. Markovića, Appl. Phys. Lett. 92, 243510 (2008).

${ }^{10} \mathrm{~S}$. Lu and B. Panchapakesan, Nanotechnology 17, 1843 (2006).

${ }^{11}$ J.-L. Sun, J. Wei, J.-L. Zhu, D. Xu, X. Liu, H. Sun, D.-H. Wu, and N.-L. Wu, Appl. Phys. Lett. 88, 131107 (2006).

${ }^{12}$ D.-H. Lien, W.-K. Hsu, H.-W. Zan, N.-H. Tai, and C.-H. Tsai, Adv. Mater (Weinheim, Ger.) 18, 98 (2006).

${ }^{13}$ J.-L. Sun, J. Xu, J.-L. Zhu, and B. Li, Appl. Phys. A: Mater. Sci. Process. 91, 229 (2008).

${ }^{14}$ P. M. Ajayan and J. M. Tour, Nature (London) 447, 1066 (2007).

${ }^{15}$ J. Zou, L. Liu, H. Chen, S. I. Khondaker, R. D. McCullough, Q. Huo, and L. Zhai, Adv. Mater. (Weinheim, Ger.) 20, 2055 (2008).

${ }^{16}$ H. Chen, H. Muthuraman, P. Stokes, J. Zou, J. Wang, Q. Huo, S. I. Khondaker, and L. Zhai, Nanotechnology 18, 415606 (2007).

${ }^{17}$ D. Stauffer, Introduction to the Percolation Theory (Francis and Taylor, London, 1991).

${ }^{18}$ See EPAPS Document No. E-APPLAB-94-004905 for supplementary material containing other signal to noise ratios and $I$ vs $t$ curves. For more information on EPAPS, see http://www.aip.org/pubservs/epaps.html.

${ }^{19}$ B. Pradhan, K. Setyowati, H. Liu, D. H. Waldeck, and J. Chen, Nano Lett. 8, 1142 (2008). 